\title{
PARAMETRIZATIONS OF SADDLE SURFACES, WITH APPLICATION TO THE PROBLEM OF PLATEAU $\dagger$
}

\author{
BY \\ E. J. MCSHANE $\ddagger$
}

Introduction. In the study of the properties of rectifiable curves $x=x(t)$, $y=y(t), z=z(t)$, and of integrals of the calculus of variations taken along such curves, the investigator is greatly aided by the fact that the absolute continuity of $x(t), y(t)$ and $z(t)$ is known to be necessary and sufficient in order that the length of the curve be equal to the classical integral $\int\left[x^{\prime 2}\right.$ $\left.+y^{\prime 2}+z^{\prime 2}\right]^{1 / 2} d t$, and also by the existence of a parametric representation of the curve (in terms of length of arc) in which the defining functions are Lipschitzian. On the other hand, let us suppose that the continuous surface $S$, represented by the equations $x=x(u, v), y=y(u, v), z=z(u, v)$, has finite area in the sense of Lebesgue. We know no conditions necessary and sufficient to insure that the area of $S$ be equal to $\iint\left(E G-F^{2}\right)^{1 / 2} d u d v$, nor can we in general find a parametric representation of $S$ which enjoys any particularly desirable properties. However, in a previous paper $\S$ I have found certain conditions on the functions $x(u, v), y(u, v), z(u, v)$ which are sufficient to insure that the area be given by the classical double integral; and I have shown that on the class of all surfaces satisfying these conditions the double integrals of the kind usually considered in the calculus of variations have the property of semi-continuity.

It is therefore desirable to show that large classes of surfaces can be given representations satisfying the above mentioned conditions. Certainly it is not true that all continuous surfaces can be given such representations. However, let us restrict our attention to the class of surfaces for which the defining functions $x(u, v)$, etc., are monotonic in the sense of Lebesgue (including in particular the important class of saddle surfaces $\|)$. In the present paper it is shown that for every such surface a representation can be found which satisfies the conditions mentioned, and is in fact almost as advantageous as a Lipschitzian representation would be.

$\dagger$ Presented to the Society, October 29, 1932; received by the editors July 19, 1932, and, after revision, December 20, 1932.

$\ddagger$ National Research Fellow.

$\S$ Integrals over surfaces in parametric form, Annals of Mathematics, vol. 34 .

\|The definition of the term "saddle surface" is given in $\$ 1$. 
One might readily suspect that such a representation would not be devoid of utility. As a matter of fact, a first use presents itself almost immediately; for with little additional effort we arrive at a solution $\dagger$ of the problem of Plateau, a solution not without interest when viewed as an application of general theorems proper to the direct method of the calculus of variations. To those readers who are interested principally in the problem of Plateau and only secondarily in the general theorems, I would like to point out that the distinctive feature of the present method is not its elegance (in which respect it is inferior to its predecessors) but the directness of the line of thought. First a solution of the problem of least area is found; then this solution is shown to admit of a representation which is in a sense almost everywhere conformal, so that the surface has to be minimal.

Finally, I would like to remark that whatever familiarity I may have with this branch of mathematics is due in large part to my conversations with Professor Radó.

1. Monotonic functions and saddle surfaces. Let the function $f(u, v)$ be defined and continuous on a point set $E$ consisting of an open set plus its boundary. We definet the monotonic deficiency of $f$ (as Lebesgue did) in the following manner. Let $R$ be any open set in $E$ and $R^{*}$ its boundary, and denote the maximum and minimum of $f$ on $R+R^{*}$ by $L, l$ respectively, and the maximum and minimum of $f$ on $R^{*}$ by $L_{1}, l_{1}$ respectively. Then the least upper bound of the quantities $L-L_{1}$ and $l_{1}-l$ as $R$ varies over all open sets contained in $E$ will be called the monotonic deficiency of $f$. Clearly this quantity is $\geqq 0$.

The function $f$ will be called monotonic if its monotonic deficiency is zero.

Suppose now that $S$ is a continuous surface $\S$ represented by the equations

$$
x=x(u, v), y=y(u, v), z=z(u, v),(u, v) \text { on } B,
$$

where $B$ is a region consisting of a Jordan curve and its interior. The surface $S$ is said to be a saddle surface provided that for every triple of constants $a$, $b, c$ the linear combination $a x(u, v)+b y(u, v)+c z(u, v)$ is a monotonic func-

† Previous solutions of this problem have been obtained by J. Douglas and by T. Rad6. The solutions by these authors of the problem of Plateau in its usual form are summed up in the following papers:

J. Douglas, Solution of the problem of Plateau, these Transactions, vol. 33 (1931), p. 263;

T. Rad6, The problem of the least area and the problem of Plateau, Mathematische Zeitschrift, vol. $32(1930)$, p. 763.

$\ddagger$ H. Lebesgue, Sur le problème de Dirichlet, Rendiconti del Circolo Matematico di Palermo, vol. 24 (1907), p. 385.

$\S$ We assume that the reader is familiar with the definition of distance of continuous surfaces and of Lebesgue area, as presented, e.g., by Radó (loc. cit., pp. 772-774) or McShane (Annals of Mathematics, vol. 33, pp. 461-463). 
tion. If $S$ has everywhere a well-defined total curvature, this is equivalent to requiring that the total curvature be non-positive; if $x=u$ and $y=v$, so that $z=z(x, y)$, this is equivalent to Radó's definition. $\dagger$

It is however our duty to show that the property of being a saddle surface is a property of the surface $S$, and does not depend on the particular representation of $S$. To do this we show that for every surface $S$ it is true that for every pair of representations

$$
\begin{aligned}
& x=x(u, v), y=y(u, v), z=z(u, v), \quad(u, v) \text { on } B, \\
& x=\bar{x}(\bar{u}, \bar{v}), \quad y=\bar{y}(\bar{u}, \bar{v}), \quad z=\bar{z}(\bar{u}, \bar{v}), \quad(\bar{u}, \bar{v}) \text { on } \bar{B},
\end{aligned}
$$

of the same surface $S$, and for every triple of constants $a, b, c$, the monotonic deficiency of $a x+b y+c z$ is equal to the monotonic deficiency of $a \bar{x}+b \bar{y}+c \bar{z}$. The proof of this statement offers no difficulty, and from it our assertion concerning saddle surfaces follows immediately.

However, the class of surfaces for which we shall obtain a special representation is larger than the class of saddle surfaces, and consists in fact of those surfaces for which the defining functions $x(u, v), y(u, v), z(u, v)$ are each monotonic. The point particularly to be observed is that this property is independent of the particular representation of the surface.

2. Lemma on convergence. The functions with which we shall be particularly concerned are those satisfying the following conditions.

(2.1a) The function $f(u, v)$ is defined and continuous on the unit circle $C$ : $u^{2}+v^{2} \leqq 1$.

(2.1b) $f(u, v)$ is absolutely continuous in $u$ for almost all fixed values of $v$, and absolutely continuous in $v$ for almost all fixed values of $u$.

(2.1c) The integral

$$
I(f) \equiv \iint_{u^{2}+v<<1}\left[(\partial f / \partial u)^{2}+(\partial f / \partial v)^{2}\right] d u d v
$$

exists. $\ddagger$

We can now state an extension of a theorem of Lebesgue $\$$ which is to play an important role in the following pages.

† See, e.g., Rad6, Geometrische Betrachtungen über zweidimensionale reguläre Variationsprobleme, Acta Szeged, vol. 2 (1926), pp. 228-253, especially pp. 229-230.

‡ This and all other integrals are understood to be Lebesgue integrals.

\& H. Lebesgue, loc. cit., p. 386. 
LEMMA 1. Let the functions $f_{1}(u, v), f_{2}(u, v), \cdots$ be defined and satisfy conditions (2.1) on the unit circle $C$. Suppose further that the sequence $\left\{f_{n}(u, v)\right\}$ is uniformly convergent on the circumference of $C$, and that the monotonic deficiency of $f_{n}(u, v)$ tends to zero with $1 / n$, and also that $I\left(f_{n}\right) \leqq H$ for every $n$, where $H$ is some constant. Then there exists a subsequence of the $\left\{f_{n}(u, v)\right\}$ converging uniformly on the whole circle $C$ to a monotonic limit function $f(u, v)$, and $f(u, v)$ also satisfies conditions (2.1).

To prove the existence of a uniformly convergent subsequence of $\left\{f_{n}\right\}$ we can follow the proof of Lebesgue $\dagger$ almost without change. The fact that the $f_{n}(u, v)$ are not all identical on the boundary, but merely converge uniformly, causes no trouble. But we find it convenient to replace the circles $C(r)$ used by Lebesgue in his proof by squares $Q(r)$; this device will be used in the proof of Lemma 2, and we therefore do not give it in detail here.

It remains to prove that the limit function $f(u, v)$ satisfies conditions (2 1). To do this we use a slight modification of a theorem of Fatouf: If the functions $\phi_{1}(x), \phi_{2}(x), \ldots$ are all non-negative and their integrals

$$
\int_{a}^{b} \phi_{n}(x) d x
$$

are all less than a fixed number, then $\lim$ inf $\phi_{n}(x)$ is summable, and

$$
\int_{a}^{b} \lim \inf \phi_{n}(x) d x \leqq \lim \inf \int_{a}^{b} \phi_{n}(x) d x .
$$

The proof differs only very slightly from that given by Fatou. Returning to our uniformly convergent subsequence of the functions $f_{n}(u, v)$ (for which subsequence we retain the notation $\left.\left\{f_{n}(u, v)\right\}\right)$, we find by virtue of this theorem that $\S$

$$
\int_{-1}^{1} d u \cdot \lim \inf \int d v\left(\partial f_{n} / \partial v\right)^{2} \leqq \lim \inf \int_{-1}^{1} d u \cdot \int d v\left(\partial f_{n} / \partial v\right)^{2} \leqq H .
$$

Hence the set of values of $u$ for which the expression

$$
\lim \inf \int\left(\partial f_{n} / \partial v\right)^{2} d v
$$

$\dagger$ Loc. cit.

‡ P. Fatou, Séries trigonométriques et séries de Taylor, Acta Mathematica, vol. 30 (1906), p. 375.

$\$$ All single integrals in this proof are understood to have the limits $-\left(1-u^{2}\right)^{1 / 2},+\left(1-u^{2}\right)^{1 / 2}$, unless other ranges are specifically indicated. 
is infinite has measure zero. Likewise, since $\left(\partial f_{n} / \partial u\right)^{2}$ is summable over the unit circle, the integral

$$
\int\left(\partial f_{n} / \partial v\right)^{2} d v
$$

is finite for almost all values of $u$. We define $E$ to be the set of values of $u$ for which one or more of the expressions (2.2), (2.3) is infinite; this set has measure zero, and we shall henceforward restrict our attention to values of $u$ belonging to the complement of $E$.

We now fix upon any value of $u$ belonging to the complement of $E$, and select a subsequence $f_{\alpha}(u, v)$ of our original convergent subsequence ( $\alpha$ ranges over a subset of the positive integers) for which there exists the limit

$$
\lim _{\alpha \rightarrow \infty} \int\left(\partial f_{\alpha}(u, v) / \partial v\right)^{2} d v=\lim \inf \int\left(\partial f_{n}(u, v) / \partial v\right)^{2} d v
$$

By the proof of a theorem of F. Riesz $\dagger$ it is possible to select a subsequence of the sequence $\left\{f_{\alpha}\right\}$ which converges everywhere to a function $\phi(v)$ which is absolutely continuous and whose derivative $d \phi / d v$ is summable together with its square, and in addition

$$
\int(d \phi / d v)^{2} d v \leqq \lim _{\alpha \rightarrow \infty} \int\left(\partial f_{\alpha} / \partial v\right)^{2} d v .
$$

But the sequence $\left\{f_{n}\right\}$ converges uniformly to $f(u, v)$; hence $\phi(v)=f(u, v)$, so that $f(u, v)$ is absolutely continuous in $v$ and

$$
\int(\partial f / \partial v)^{2} d v \leqq \lim \inf \int\left(\partial f_{n} / \partial v\right)^{2} d v
$$

Applying Fatou's theorem once again, we obtain

$$
\begin{gathered}
\int_{C E} d u \int d v(\partial f / \partial v)^{2} \leqq \int_{C E} d u \cdot \lim \inf \int d v\left(\partial f_{n} / \partial v\right)^{2} \\
\leqq \lim \inf \iint_{u^{2}+v^{2}<1}\left(\partial f_{n} / \partial v\right)^{2} d u d v \leqq H .
\end{gathered}
$$

Likewise $f(u, v)$ is absolutely continuous in $u$ for almost all fixed values of $v$, and

$$
\iint_{u^{2}+v<<1}(\partial f / \partial u)^{2} d u d v \leqq \lim \inf \iint_{u^{2}+v^{2}<1}\left(\partial f_{n} / \partial u\right)^{2} d u d v \leqq H
$$

$\dagger$ F. Riesz, Untersuchungen über Systeme integrierbarer Funktionen, Mathematische Annalen, vol. 69 (1910), pp. 466-468. 
Adding inequalities (2.4) and (2.5), we find that $I(f)$ exists and moreover

$$
I(f) \leqq \lim \inf I\left(f_{n}\right) .
$$

3. Convergence on the boundary. For the sake of compactness, the boundary curve

$$
x=x(\cos \theta, \sin \theta), y=y(\cos \theta, \sin \theta), z=z(\cos \theta, \sin \theta)
$$

of the surface

$$
x=x(u, v), y=y(u, v), z=z(u, v), u^{2}+v^{2} \leqq 1,
$$

will be written in the shorter form

$$
x=x(\theta), y=y(\theta), z=z(\theta) .
$$

The symbols $E, F, G$ will as usual have the respective meanings $x_{u}^{2}+y_{u}^{2}+z_{u}^{2}$, $x_{u} x_{v}+y_{u} y_{v}+z_{u} z_{v}, x_{v}^{2}+y_{v}^{2}+z_{v}^{2}$.

We now proceed to prove our second lemma.

Lemma 2. Let $\left\{S_{n}\right\}$ be a sequence of surfaces possessing representations

$$
x=x_{n}(u, v), y=y_{n}(u, v), z=z_{n}(u, v), u^{2}+v^{2} \leqq 1,
$$

in which the functions $x_{n}, y_{n}, z_{n}$ satisfy conditions (2.1), and for which

$$
\iint_{u s+v<1}\left(E_{n}+G_{n}\right) d u d v \leqq H
$$

for all values of $n, H$ being a fixed number. Suppose further that the boundary curves

$$
\Gamma_{n}: \quad x=x_{n}(\theta), y=y_{n}(\theta), z=z_{n}(\theta)
$$

of the surfaces $S_{n}$ approach as a limit a simple closed curve $\Gamma$, and that for three distinct values $\theta_{1}, \theta_{2}, \theta_{3}$ of $\theta$ the sequences of points $\left\{x_{n}\left(\theta_{i}\right), y_{n}\left(\theta_{i}\right), z_{n}\left(\theta_{i}\right)\right\} a p-$ proach three distinct limit points $\left(\xi_{i}, \eta_{i}, \zeta_{i}\right)(i=1,2,3)$. Then it is possible to find a representation

$$
x=x(\theta), y=y(\theta), z=z(\theta)
$$

of the curve $\Gamma$ and a subsequence of the $\left\{S_{n}\right\}$ (for which we retain the same notation) such that

$$
\lim x_{n}(\theta)=x(\theta), \quad \lim y_{n}(\theta)=y(\theta), \quad \lim z_{n}(\theta)=z(\theta)
$$

uniformly in $\theta$.

† My original proof of this lemma was decidedly more intricate than the above; for the present proof I wish here to thank Professor Tamarkin. 
Let $\left\{\epsilon_{n}\right\}$ be a sequence of positive numbers tending to zero such that the distance $\left\|\Gamma_{n}, \Gamma\right\|$ between $\Gamma_{n}$ and $\Gamma$ is less than $\epsilon_{n}$ for every $n$. If we fix upon any topological representation $x=x(\tau), y=y(\tau), z=z(\tau)$ of the curve $\Gamma$ on the unit circumference (the functions $x, y, z$ having period $2 \pi$ ), then we can find a topological mapping of the unit circumference on itself, expressible by the equation $\tau=\tau_{n}(\theta), 0 \leqq \theta \leqq 2 \pi$, in which $\tau_{n}(\theta)$ is a continuous monotonic function such that $0 \leqq \tau(0)=\tau_{n}(2 \pi)-2 \pi \leqq 2 \pi$, for which

$$
\left|x_{n}(\theta)-x\left(\tau_{n}(\theta)\right)\right|<\epsilon_{n},
$$

with like inequalities for $y$ and $z$. By Helly's theorem there exists a subsequence of the sequence $\left\{\tau_{n}(\theta)\right\}$ which converges for each $\theta$ in the interval $(0,2 \pi)$ to a monotonic limit function $\tau(\theta)$. We reject all $\tau_{n}$ and their corresponding $S_{n}$ which do not belong to this subsequence, and we re-name the remaining subsequence $\left\{\tau_{n}\right\}$, and the remaining surfaces $\left\{S_{n}\right\}$.

We thus find that for every $\theta$ we have $\lim \tau_{n}(\theta)=\tau(\theta)$; and since $x(\tau)$, $y(\tau), z(\tau)$ are continuous, this implies $\lim x\left(\tau_{n}(\theta)\right)=x(\tau(\theta))$ for every $\theta$. From this and inequality (3.4) it follows that for every $\theta$ we have

$$
\lim x_{n}(\theta)=x(\tau(\theta)) ;
$$

similar equations hold for $y$ and $z$. In particular, for the values $\theta_{1}, \theta_{2}, \theta_{3}$ of the hypothesis we have $\lim x_{n}\left(\theta_{i}\right)=\xi_{i}$, whence $x\left(\tau\left(\theta_{i}\right)\right)=\xi_{i}$; likewise $y\left(\tau\left(\theta_{i}\right)\right)$ $=\eta_{i}, z\left(\tau\left(\theta_{i}\right)\right)=\zeta_{i}$. Since the three points $\left(\xi_{i}, \eta_{i}, \zeta_{i}\right)$ are distinct, the three numbers $\tau\left(\theta_{i}\right)$ must also be distinct.

We now prove that the function $\tau(\theta)$ is continuous. Suppose on the contrary that there is a point $\dagger \theta_{0}$ of discontinuity of $\tau(\theta)$; then $\tau\left(\theta_{0}-0\right)$ and $\tau\left(\theta_{0}+0\right)$ both exist, and $\tau\left(\theta_{0}+0\right)>\tau\left(\theta_{0}-0\right)$. We cannot have $\tau\left(\theta_{0}+0\right)$ $=\tau\left(\theta_{0}-0\right)+2 \pi$; for this would imply that $\tau(\theta)$ is constantly equal to $\tau\left(\theta_{0}-0\right)$ on the interval $\left(0, \theta_{0}\right)$, and constantly equal to $\tau\left(\theta_{0}+0\right)$ on the interval $\left(\theta_{0}, 2 \pi\right)$, as readily follows from the fact that $\tau(\theta)$ is monotonic and $\tau(2 \pi)=\tau(0)+2 \pi$. This is in contradiction with the previously established fact that $\tau\left(\theta_{1}\right), \tau\left(\theta_{2}\right)$, and $\tau\left(\theta_{3}\right)$ are all distinct. Hence $0<\tau\left(\theta_{0}+0\right)-\tau\left(\theta_{0}-0\right)$ $<2 \pi$; and since the equations $x=x(\tau)$, etc., map the unit circumference topologically on $\Gamma$, the points

$$
\left(x\left(\tau\left(\theta_{0}-0\right)\right), y\left(\tau\left(\theta_{0}-0\right)\right), z\left(\tau\left(\theta_{0}-0\right)\right)\right)
$$

and

$$
\left(x\left(\tau\left(\theta_{0}+0\right)\right), y\left(\tau\left(\theta_{0}+0\right)\right), z\left(\tau\left(\theta_{0}+0\right)\right)\right)
$$

are distinct. Suppose to be specific that

$\dagger$ The following proof is constructed for the case in which $\theta_{0}$ is an interior point of the interval $(0,2 \pi)$. In case $\theta_{0}$ is an end point of the interval, say $\theta_{0}=0$, we have only to remember that $\tau(\theta)$ and all the $\tau_{n}(\theta)$ are periodic, and consider them as defined on the interval $(-\pi, \pi)$. 


$$
x\left(\tau\left(\theta_{0}+0\right)\right)=x\left(\tau\left(\theta_{0}-0\right)\right)+3 \epsilon, \epsilon>0 .
$$

Since $x(\tau)$ is continuous, there exists a positive number $\delta(<\pi / 3)$ such that

$$
\left|x(\tau(\theta))-x\left(\tau\left(\theta_{0}-0\right)\right)\right|<\epsilon \quad\left(\theta_{0}-\delta<\theta<\theta_{0}\right)
$$

and

$$
\left|x(\tau(\theta))-x\left(\tau\left(\theta_{0}+0\right)\right)\right|<\epsilon \quad\left(\theta_{0}<\theta<\theta_{0}+\delta\right) .
$$

We now introduce a new coordinate system convenient for our present purposes. Let $Q(r)$ be the square (the line-configuration, not the region) with center at the point $\left(\cos \theta_{0}, \sin \theta_{0}\right)$ and sides of length $2 r$ parallel to the coordinate axes. If $4 r \leqq \delta$, the square $Q(r)$ intersects the circumference once in the interval $\left(\theta_{0}-\delta, \theta_{0}\right)$ and once in the interval $\left(\theta_{0}, \theta_{0}+\delta\right)$. To each point $(u, v)$ on $Q(r)$ we assign the coordinates $(r, s)$, where $s$ is the length of arc of $Q(r)$ from the point at which $Q(r)$ enters the circle $C$ to the point $(u, v)$, measured counter-clockwise. Then $\left(\partial x_{n}(r, s) / \partial s\right)^{2}$ is equal to $\left(\partial x_{n}(u, v) / \partial u\right)^{2}$ or to $\left(\partial x_{n}(u, v) / \partial v\right)^{2}$, according to the side of $Q(r)$ on which $(u, v)$ lies; and therefore denoting by $s(r)$ the $s$-coordinate of the point at which $Q(r)$ leaves the circle, we have

$$
\begin{aligned}
\int_{0}^{\delta / 4} \int_{0}^{s(r)}\left(\partial x_{n}(r, s) / \partial s\right)^{2} d s d r & \\
\leqq & \leqq \int_{u^{2}+v<<1}\left\lfloor\left(\partial x_{n} / \partial u\right)^{2}+\left(\partial x_{n} / \partial v\right)^{2}\right] d u d v \leqq H .
\end{aligned}
$$

Hence for almost all values of $r \leqq \delta / 4$ all the integrals

$$
\int_{0}^{s(r)}\left(\partial x_{n} / \partial s\right)^{2} d s
$$

are finite; and by the theorem of Fatou $\dagger$

$$
\int_{0}^{\delta / 4} \lim \inf \int_{0}^{s(r)}\left(\partial x_{n} / \partial s\right)^{2} d s \leqq \lim \inf \int_{0}^{\delta / 4} \int_{0}^{s(r)}\left(\partial x_{n} / \partial s\right)^{2} d s d r \leqq H
$$

so that for almost all values of $r \leqq \delta / 4$ the expression

$$
\lim \inf \int_{0}^{s(r)}\left(\partial x_{n} / \partial s\right)^{2} d s
$$

is finite. We define $E$ to be the set of values of $r$ for which one or more of the expressions (3.10) or (3.12) is infinite; then $E$ has measure zero. We henceforth consider only values of $r$ which belong to the complement of $E$, and

$\dagger$ I.e., as stated in the proof of Lemma 1. 
which satisfy the additional condition that all the functions $x_{n}(r, s)$ are absolutely continuous in $s$; the set of values of $r$ thus rejected has measure zero.

To the integrals (3.10) we now apply the inequality of Schwarz, thus finding

$$
\begin{gathered}
\int_{0}^{s(r)}\left(\partial x_{n} / \partial s\right)^{2} d s \geqq\left\{\int_{0}^{s(r)}\left(\partial x_{n} / \partial s\right) d s\right\}^{2} \cdot \frac{1}{s(r)} \\
=\left[x_{n}(r, s(r))-x_{n}(r, 0)\right]^{2} / s(r) .
\end{gathered}
$$

Inequalities (3.13) and (3.11) together imply that

$$
\int_{0}^{\delta / 4} \lim \inf \left[x_{n}(r, s(r))-x_{n}(r, 0)\right]^{2} \cdot \frac{1}{s(r)} \cdot d r \leqq H .
$$

But the points $(r, 0)$ and $(r, s(r))$ are both on the circumference of the circle $C$, and lie each in one of the intervals $\left(\theta_{0}-\delta, \theta_{0}\right)$ and $\left(\theta_{0}, \theta_{0}+\delta\right)$; hence by (3.6), (3.7), and (3.8) we have

$$
\lim \inf \left[x_{n}(r, s(r))-x_{n}(r, 0)\right]^{2} \geqq \epsilon^{2} .
$$

Also by its definition $s(r)<8 r$. Hence

$$
\lim \inf \left[x_{n}(r, s(r))-x_{n}(r, 0)\right]^{2} \cdot \frac{1}{s(r)}>\frac{\epsilon^{2}}{8 r},
$$

which is not summable over the interval $0 \leqq r \leqq \delta / 4$. This contradicts inequality (3.14), and therefore the assumption that $\tau(\theta)$ is discontinuous leads to a contradiction.

Therefore the sequence $\left\{\tau_{n}(\theta)\right\}$ of continuous monotonic functions converges everywhere to the continuous function $\tau(\theta)$. It follows $\dagger$ that the convergence of $\tau_{n}(\theta)$ to $\tau(\theta)$ is uniform; hence $\lim x\left(\tau_{n}(\theta)\right)=x(\tau(\theta))$ uniformly. This with (3.4) implies that $\lim x_{n}(\theta)=x(\tau(\theta))$ uniformly in $\theta$; similar statements hold for $y, z$. Hence the curve

$$
x=x(\tau(\theta)), y=y(\tau(\theta)), z=z(\tau(\theta))
$$

is a limit curve of the sequence $\left\{\Gamma_{n}\right\}$. But $\left\{\Gamma_{n}\right\}$ has the unique limit $\Gamma$; therefore equations (3.17) form a representation of $\Gamma$, and the lemma is established.

4. A theorem on representations. We now proceed to prove our principal theorem concerning parametric representations.

$\dagger$ Buchanan, H. E., and Hildebrandt, T. H., Note on the convergence of a sequence of functions of a certain type, Annals of Mathematics, vol. 9 (1908), p. 123. 
THEOREM I. If the continuous surface $S$, represented by the equations

$$
x=\bar{x}(u, v), y=\bar{y}(u, v), z=\bar{z}(u, v), u^{2}+v^{2} \leqq 1,
$$

satisfies the conditions

(4.1) the Lebesgue area $L(S)$ of the surface $S$ is finite;

(4.2) the curve

$$
\Gamma: x=\bar{x}(\theta), y=\bar{y}(\theta), z=\bar{z}(\theta)
$$

bounding $S$ is a Jordan curve;

(4.3) the functions $\bar{x}(u, v), \bar{y}(u, v), \bar{z}(u, v)$ are monotonic;

then there exists a representation

$$
x=x(u, v), y=y(u, v), z=z(u, v), u^{2}+v^{2} \leqq 1,
$$

of $S$ in which the functions $x(u, v)$, etc., satisfy the conditions (2.1), and also satisfy the relations

$$
E=G, F=0 \text { for almost all values of }(u, v) .
$$

Moreover under any change of parameters $u=u(\bar{u}, \bar{v}), v=v(\bar{u}, \bar{v})$ representing $a$ conformal mapping of the unit circle on itself the three functions $x(u(\bar{u}, \bar{v})$, $v(\bar{u}, \bar{v}))$, etc., also satisfy conditions $(2.1)$ and $(4,4)$.

Before proceeding to the proof of this theorem, we observe that the hypotheses are independent of the representation of $S$.

Now let $\left\{\Pi_{n}\right\}$ be a sequence of polyhedra tending to $S$ for which the areas $L\left(\Pi_{n}\right)$ tend to $L(S)$; we can assume without loss of generality that none of the triangles which form the faces of $\Pi_{n}$ àre degenerate. If $\Pi_{n}$ has the representation

$$
x=\bar{x}_{n}(\alpha, \beta), y=\bar{y}_{n}(\alpha, \beta), z=\bar{z}_{n}(\alpha, \beta), \alpha^{2}+\beta^{2} \leqq 1,
$$

then there exists for each $n$ a topological mapping $\alpha=\alpha_{n}(u, v), \beta=\beta_{n}(u, \dot{v})$ of the unit circle on itself such that $\lim \bar{x}_{n}\left(\alpha_{n}(u, v), \beta_{n}(u, v)\right)=\bar{x}(u, v)$ uniformly; and since $\bar{x}(u, v)$ is monotonic, this implies $\dagger$ that the monotonic deficiency of $\bar{x}_{n}\left(\alpha_{n}(u, v), \beta_{n}(u, v)\right)$ tends to zero with $1 / n$; similar statements hold for $y$ and for $z$. But the functions $\bar{x}_{n}\left(\alpha_{n}(u, v), \beta_{n}(u, v)\right)$, etc., form a representation of $\Pi_{n}$; hence by $\S 1$ the monotonic deficiency of $\bar{x}_{n}(\alpha, \beta)$ is equal to the monotonic deficiency of $\bar{x}_{n}\left(\alpha_{n}(u, v), \beta_{n}(u, v)\right)$, and consequently tends to zero with $1 / n$.

On the circumference of the unit circle we now choose three distinct points $A_{1}{ }^{*}, A_{2}{ }^{*}, A_{3}{ }^{*}$, and on the curve we choose three distinct points $A_{1}, A_{2}, A_{3}$.

† Lebesgue, loc. cit., p. 385. 
Since $\lim \Pi_{n}=S$, the boundary curves $\Gamma_{n}$ of the polyhedra $\Pi_{n}$ tend to the curve $\Gamma$; hence on each $\Gamma_{n}$ we can choose three distinct points $A_{1}^{(n)}, A_{2}^{(n)}, A_{3}^{(n)}$ such that $A_{i}^{(n)}$ approaches $A_{i}$ as $n$ increases.

From general theorems on the conformal maps of abstract Riemann surfaces $\dagger$ it follows that any polyhedron $\Pi$ whose faces are non-degenerate admits of a parametric representation of the following kind.

(a) The functions representing $\Pi$ are defined on the unit circle; i.e., $\Pi$ is given by the equations

$$
x=x(u, v), y=y(u, v), z=z(u, v), u^{2}+v^{2} \leqq 1 .
$$

(b) The unit circle is subdivided by arcs into a finite number of curvilinear triangles $\delta_{1}, \cdots, \delta_{k}$, and the equations (4.5) carry each $\delta_{i}$ in a topological way into a rectilinear triangle in $x y z$-space.

(c) The triangles $\delta_{i}$ are bounded by arcs which are analytic, including end points.

(d) Interior to each triangle $\delta_{i}$ the functions $x(u ; v), y(u, v), z(u, v)$ are analytic and satisfy the relations

$$
E=G, F=0 .
$$

(e) Three arbitrarily given distinct points $A_{1}, A_{2}, A_{3}$ on the boundary curve of $\Pi$ correspond under equations (4.5) to three arbitrarily given distinct points $A_{1}{ }^{*}, A_{2}{ }^{*}, A_{3}{ }^{*}$ on the unit circle $u^{2}+v^{2}=1$.

For such a representation of $\Pi$ we find without difficulty that conditions (2.1) are satisfied. We now choose for each $\Pi_{n}$ a representation

$$
\Pi_{n}: x=x_{n}(u, v), y=y_{n}(u, v), z=z_{n}(u, v), u^{2}+v^{2} \leqq 1,
$$

satisfying the above conditions; in particular, for the points $A_{1}{ }^{*}, A_{2}{ }^{*}, A_{3}{ }^{*}$ we choose the points already so named on the circumference of the unit circle, and for the points $A_{1}, A_{2}, A_{3}$ we choose the points $A_{1}^{(n)}, A_{2}^{(n)}, A_{3}^{(n)}$.

We now make use of the theorem that if the functions $x(u, v), y(u, v)$, $z(u, v), u^{2}+v^{2} \leqq 1$, satisfy conditions (2.1), then the area of the surface $S$ : $x=x(u, v), y=y(u, v), z=z(u, v), u^{2}+v^{2} \leqq 1$, is equal to

† See, for instance, Carathéodory, Conformal Representation (No. 28 of the Cambridge Tracts in Mathematics and Mathematical Physics), in particular chapter VII. While the theorem on the conformal maps of polyhedra can be obtained as a special case of general facts, it should be observed that this theorem was proved by H. A. Schwarz.

$\ddagger$ E. J. McShane, loc. cit. in introduction. This theorem has also been established independently and by different methods by C. B. Morrey, in a paper not as yet published.

This theorem is needed later, but for the present case it is stronger than necessary; equation (4.8) can be established by simpler means. Cf. Rad6, loc. cit., p. 774 . 


$$
\iint_{u^{2}+v^{2}<1}\left(E G-F^{2}\right)^{1 / 2} d u d v
$$

Applying this to the polyhedra $\Pi_{n}$, we find

$$
L\left(\Pi_{n}\right)=\iint_{u^{2}+v<<1}\left(E_{n} G_{n}-F_{n}^{2}\right)^{1 / 2} d u d v ;
$$

and by (4.6) this implies

$$
L\left(\Pi_{n}\right)=\iint_{u^{2}+v^{2}<1} \frac{1}{2}\left(E_{n}+G_{n}\right) d u d v .
$$

Since $\lim L\left(\Pi_{n}\right)=L(S)$, the right member of (4.9) is bounded. Observing that the equation $\lim \Pi_{n}=S$ implies that the boundary curves $\Gamma_{n}$ of the polyhedra $\Pi_{n}$ converge to $\Gamma$, we find that all of the hypotheses of Lemma 2 are satisfied. We can therefore select a representation $x=x(\theta), y=y(\theta), z=z(\theta)$ of $\Gamma$ and a subsequence of the $\left\{\Pi_{n}\right\}$ (for which we retain the same notation) such that

$$
\lim x_{n}(\theta)=x(\theta), \lim y_{n}^{*}(\theta)=y(\theta), \lim z_{n}(\theta)=z(\theta)
$$

uniformly in $\theta$.

This subsequence now satisfies the hypotheses of Lemma 1, and we can therefore select from it a subsequence (which we continue to call $\left\{\Pi_{n}\right\}$ ) for which the functions $x_{n}(u, v), y_{n}(u, v), z_{n}(u, v)$ converge uniformly on the whole circle to limit functions $x(u, v), y(u, v), z(u, v)$ which satisfy conditions (2.1). Therefore the surface defined by the equations

$$
x=x(u, v), y=y(u, v), z=z(u, v), u^{2}+v^{2} \leqq 1,
$$

is a limit surface of the sequence $\left\{\Pi_{n}\right\}$. But the sequence has the unique limit $S$; hence equations (4.11) form a new representation of the surface $S$.

Since the functions $x(u, v), y(u, v), z(u, v)$ satisfy conditions $(2.1)$, the area is given by the classical integral; hence

$$
\begin{aligned}
\iint_{u^{2+v}<1} \frac{1}{2}(E+G) d u d v & \geqq \iint_{u^{3}+v^{2}<1}(E G)^{1 / 2} d u d v \\
& \geqq \iint_{u^{2}+v<<1}\left(E G-F^{2}\right)^{1 / 2} d u d v=L(S) .
\end{aligned}
$$

On the other hand, we know by (4.9) and (2.6) that

(4.13) $\lim L\left(\Pi_{n}\right)=\lim \iint_{u^{2}+v^{2}<1} \frac{1}{2}\left(E_{n}+G_{n}\right) d u d v \geqq \iint_{u^{2}+v^{2<1}} \frac{1}{2}(E+G) d u d v$; and since $\lim L\left(\Pi_{n}\right)=L(S)$, these inequalities imply 


$$
L(S)=\iint_{u^{2}+v<1} \frac{1}{2}(E+G) d u d v=\iint_{u^{2}+v<<1}\left(E G-F^{2}\right)^{1 / 2} d u d v .
$$

Now $(E+G) / 2$ is never less than $\left(E G-F^{\prime}\right)^{\prime}: / 2$, and if $E, F$ and $G$ are finite the two can be equal only if $E=G$ and $F=0$. Hence from (4.14) it follows that

$$
E=G, F=0 \text { for almost all values of }(u, v) \text {. }
$$

It remains only to show that these properties ((2.1) and (4.15)) of $x(u, v)$, $y(u, v), z(u, v)$ remain invariant under conformal mappings

$$
u=u(\bar{u}, \bar{v}), v=v(\bar{u}, \bar{v})
$$

of the unit circle on itself. We retain the sequence of polyhedra $\left\{\Pi_{n}\right\}$ obtained above for which the representing functions $x_{n}(u, v), y_{n}(u, v), z_{n}(u, v)$ are uniformly convergent. Apply to each of these the transformation (4.16); we obtain a new representation

$$
x=\bar{x}_{n}(\bar{u}, \bar{v}) \equiv x_{n}(u(\bar{u}, \bar{v}), v(\bar{u}, \bar{v})), y=\bar{y}_{n}(\bar{u}, \bar{v}), z=\bar{z}_{n}(\bar{u}, \bar{v}) .
$$

Since the mapping (4.16) is conformal, the functions $\bar{x}_{n}(\bar{u}, \bar{v})$, etc., continue to satisfy all the conditions (a), . , (e) stated above. Also the sequences $\bar{x}_{n}(\bar{u}, \bar{v})$, etc., are uniformly convergent; let the limit function be $\bar{x}(\bar{u}, \bar{v})$. Then by the definition (4.17) of $\bar{x}_{n}(\bar{u}, \bar{v})$, we have $\bar{x}(\bar{u}, \bar{v})=x(u(\bar{u}, \bar{v}), v(\bar{u}, \bar{v}))$, with like equations for $y$ and $z$. All the arguments of the preceding paragraph are applicable, and we thus find that $\bar{x}(\bar{u}, \bar{v})$, etc., also satisfy conditions (2.1) and (4.4). The theorem is thus established.

5. An area-reducing alteration. We shall in studying the problem of Plateau have need of one further lemma.

\section{LEMMA 3. Let the continuous surface}

$$
S: x=x(u, v), y=y(u, v), z=z(u, v), u^{2}+v^{2} \leqq 1,
$$

have finite area: Then there exists a surface $\bar{S}: x=\bar{x}(u, v), y=\bar{y}(u, v), z=\bar{z}(u, v)$, $u^{2}+v^{2} \leqq 1$, having the same boundary curve as $S$, and satisfying the conditions

$$
L(\bar{S}) \leqq L(S),
$$

(5.3) the functions $\bar{x}(u, v), \bar{y}(u, v), \bar{z}(u, v)$ are monotonic.

We need only a slight modification of a proof due to Lebesgue. $\dagger$ Let us arrange the rational numbers in a sequence $r_{1}, r_{2}, \ldots$. The point set at which $x(u, v)>r_{1}$ is (if not null) an openł set, and consists of a finite or denumerable set of maximal open connected sets. We disregard those sets

$\dagger$ Loc. cit., p. 382.

$\ddagger$ Except that it may contain limit points on the circumference. 
which have points in common with the circumference $u^{2}+v^{2}=1$, and name the remainder $R_{1}, R_{2}, \cdots$. We treat similarly the point set for which $x(u, v)<r_{1}$; the maximal connected open point sets, interior to the circle, on which $x(u, v)<r_{1}$ we call $T_{1}, T_{2}, \cdots$. We designate by $x^{(1)}(u, v)$ the function equal to $r_{1}$ on $R_{1}+T_{1}+R_{2}+T_{2}+\cdots$, and equal to $x(u, v)$ elsewhere; this is a continuous function. Moreover, the area of the surface

$$
S^{(1)}: \quad x=x^{(1)}(u, v), y=y(u, v), z=z(u, v)
$$

is at most equal to $L(S)$. This is obvious if $S$ is a polyhedron, for then the images of $R_{1}, T_{1}$, etc., under (5.1) consist of a finite number of triangles, and the images under (5.4) are the projections of those triangles on the plane $x=r_{1}$. For the general case, we select (as is always possible) a sequence $\left\{\Pi_{n}\right\}$ of polyhedra represented in the form

$$
x=x_{n}(u, v), y=y_{n}(u, v), z=z_{n}(u, v), u^{2}+v^{2} \leqq 1,
$$

such that $\lim L\left(\Pi_{n}\right)=L(S)$, and $\lim x_{n}(u, v)=x(u, v)$ uniformly on the circle, with similar statements for $y$ and $z$. We obtain $x_{n}^{(1)}(u, v)$ from $x_{n}(u, v)$ as above, and denote by $\Pi_{n}^{(1)}$ the polyhedron

$$
x=x_{n}^{(1)}(u, v), y=y_{n}(u, v), z=z_{n}(u, v), u^{2}+v^{2} \leqq 1 .
$$

Then $\lim \Pi_{n}^{(1)}=S^{(1)}$, and $L\left(\Pi_{n}^{(1)}\right) \leqq L\left(\Pi_{n}\right)$; hence

$$
L\left(S^{(1)}\right) \leqq \lim \inf L\left(\Pi_{n}^{(1)}\right) \leqq \lim L\left(\Pi_{n}\right)=L(S) .
$$

We now obtain $x^{(2)}(u, v)$ from $x^{(1)}(u, v)$ as we obtained $x^{(1)}(u, v)$ from $x(u, v)$, the number $r_{2}$ taking the place of $r_{1}$. For the corresponding surface $S^{(2)}$ we have $L\left(S^{(2)}\right) \leqq L(S)$. Likewise we obtain $x^{(3)}$ from $x^{(2)}$, using $r_{3}$ instead of $r_{2}$; and so on. The sequence of functions $\left\{x^{(n)}(u, v)\right\}$ converges uniformly to a limit function $\bar{x}(u, v)$, which is continuous and monotonic; the proof of this is identical with that given by Lebesguet, and we shall not repeat it. We wish to emphasize two points; first, the functions $x^{(n)}(u, v)$ are all equal to $x(u, v)$ on the circumference $u^{2}+v^{2}=1$, so that $\bar{x}(\theta)=x(\theta)$; and second, the surface $S_{x}$ defined by the equations $x=\bar{x}(u, v), y=y(u, v)$, $z=z(u, v)$ has area at most equal to $L(S)$, since

$$
L\left(S_{x}\right) \leqq \lim \inf L\left(S^{(n)}\right) \leqq L(S) .
$$

The whole process being repeated for the function $y(u, v)$, we obtain a monotonic function $\bar{y}(u, v)$, equal to $y(u, v)$ on the circumference, and such that the surface defined by the equations $x=\bar{x}(u, v), y=\bar{y}(u, v), z=z(u, v)$ has area at most equal to $L(S)$. Finally we repeat the whole process for

$\dagger$ Loc. cit.; beginning at the middle of p. 382. 
$z(u, v)$, and obtain a monotonic function $\bar{z}(u, v)$, equal to $z(u, v)$ on the circumference $u^{2}+v^{2}=1$, and such that the surface $\bar{S}$ defined by the equations

$$
x=\bar{x}(u, v), y=\bar{y}(u, v), z=\bar{z}(u, v), u^{2}+v^{2} \leqq 1,
$$

has area $L(\bar{S}) \leqq L(S)$. The lemma is thus established.

6 . The problem of Plateau. Let $\Gamma$ be any Jordan curve in $x y z$-space; we designate by $a(\Gamma)$ the greatest lower bound of the areas of all continuous surfaces $S$ bounded by $\Gamma$. On the other hand, let $\left\{\Pi_{n}\right\}$ be a sequence of polyhedra whose boundary curves tend to $\Gamma$, and consider the quantity lim inf $L\left(\Pi_{n}\right)$. The greatest lower bound of this quantity for all such sequences $\left\{\Pi_{n}\right\}$ is called the minimum area of $\Gamma$; we denote it by $m(\Gamma)$. There is no essential restriction in assuming that each polyhedron $\Pi_{n}$ of the sequence be bounded by a Jordan polygon; for given $\Pi_{n}$, we can alter it so as to make the boundary non-self-intersecting while changing the area and displacing the boundary by arbitrarily small amounts.

It is easy to show that

$$
m(\Gamma) \leqq a(\Gamma) .
$$

For let $S$ be any continuous surface bounded by $\Gamma$, and let $\left\{\Pi_{n}\right\}$ be a sequence of polyhedra tending to $S$ and such that $L\left(\Pi_{n}\right)$ tends to $L(S)$. The boundaries $\Gamma_{n}$ of the polyhedra $\Pi_{n}$ tend to $\Gamma$, and therefore

$$
m(\Gamma) \leqq \lim \inf L\left(\Pi_{n}\right)=L(S) ;
$$

this being true for every surface $S$ bounded by $\Gamma$, inequality (6.1) follows immediately. We shall later prove (as is already known $\dagger$ ) that $a(\Gamma)=m(\Gamma)$ for every Jordan curve $\Gamma$.

We now proceed to prove

THEOREM $\ddagger$ II. For every Jordan curve $\Gamma$ whose minimum area $m(\Gamma)$ is finite, there exists a continuous surface

$$
S: x=x(u, v), y=y(u, v), z=z(u, v), u^{2}+v^{2} \leqq 1,
$$

bounded by $\Gamma$ and satisfying the following conditions:

(a) the area of $S$ is the least possible among all surfaces bounded by $\Gamma$, i.e.,

$$
L(S)=a(\Gamma)=m(\Gamma) ;
$$

(b) the functions $x(u, v), y(u, v)$, and $z(u, v)$ are analytic, and in fact harmonic, for $u^{2}+v^{2}<1$;

(c) the surface $S$ is a minimal surface.

† Rad6, loc. cit., p. 776.

$\ddagger$ Douglas, loc. cit.; Radó, loc. cit., p 791 
Let $\left\{\Pi_{n}\right\}$ be a sequence of polyhedra having areas $L\left(\Pi_{n}\right)$ tending to $m(\Gamma)$, and bounded by Jordan polygons $\Gamma_{n}$ tending to $\Gamma$. By Lemma 3 , for each $n$ we can find a surface $S_{n}$ bounded by $\Gamma_{n}$ and of area $L\left(S_{n}\right) \leqq L\left(\Pi_{n}\right)$ for which the representing functions $\bar{x}_{n}(u, v), \bar{y}_{n}(u, v), \bar{z}_{n}(u, v)$ are monotonic. Now let $A_{1}{ }^{*}, A_{2}{ }^{*}, A_{3}{ }^{*}$ be three distinct points on the unit circumference $u^{2}+v^{2}=1$, and let $A_{1}, A_{2}, A_{3}$ be three distinct points on $\Gamma$. Since $\Gamma_{n}$ tends to $\Gamma$, we can on each $\Gamma_{n}$ select three points $A_{1}^{(n)}, A_{2}^{(n)}, A_{3}^{(n)}$ such that $A_{i}^{(n)}$ tends to $A_{i}(i=1,2,3)$. By Theorem I there exists a representation

$$
x=x_{n}(u, v), y=y_{n}(u, v), z=z_{n}(u, v), u^{2}+v^{2} \leqq 1,
$$

of $S_{n}$ such that the functions $x_{n}(u, v)$, etc., satisfy conditions (2.1), and

$$
E_{n}=G_{n} \text { and } F_{n}=0 \text { almost everywhere. }
$$

Moreover, we may assume that equations (6.4) carry the points $A_{1}{ }^{*}, A_{2}{ }^{*}, A_{3}{ }^{*}$ into $A_{1}^{(n)}, A_{2}^{(n)}, A_{3}^{(n)}$ respectively; for if the $A_{i}^{(n)}$ correspond under (6.4) to $B_{i}^{*}$ $(i=1,2,3)$ on the unit circle, then by a conformal mapping $u=u(\bar{u}, \bar{v})$, $v=v(\bar{u}, \bar{v})$, we can map the $B_{i}{ }^{*}$ on the $A_{i}{ }^{*}$, and by Theorem I the new functions $x(u(\bar{u}, \bar{v}), v(\bar{u}, \bar{v}))$, etc., continue to satisfy conditions (2.1) and (6.5). By (6.3), we have

$$
\begin{aligned}
\iint_{u^{3+v}<1} \frac{1}{2}\left(E_{n}+G_{n}\right) d u d v \\
=\iint_{u^{2}+v<1}\left(E_{n} G_{n}-F_{n}^{2}\right)^{1 / 2} d u d v=L\left(S_{n}\right) \leqq L\left(\Pi_{n}\right) ;
\end{aligned}
$$

hence the first expression in (6.6) is bounded. The sequence $\left\{S_{n}\right\}$ therefore satisfies the hypotheses of Lemma 2 , and so there exists a representation $x=x(\theta), y=y(\theta), z=z(\theta)$ of the curve $\Gamma$ and a subsequence of $\left\{S_{n}\right\}$ (for which we retain the same notation) such that

$$
\lim x_{n}(\theta)=x(\theta), \quad \lim y_{n}(\theta)=y(\theta), \quad \lim z_{n}(\theta)=z(\theta)
$$

uniformly in $\theta$.

The surface $S_{n}$ has one representation $x=\bar{x}_{n}(u, v)$, etc., in which the functions $\bar{x}_{n}(u, v)$, etc., are monotonic; hence by $\$ 1$ we know that in the representation (6.4) of $S_{n}$ the functions $x_{n}(u, v)$, etc., are monotonic. The sequences $\left\{x_{n}(u, v)\right\},\left\{y_{n}(u, v)\right\},\left\{z_{n}(u, v)\right\}$ are therefore seen to satisfy all the hypotheses of Lemma 1 ; hence there exist monotonic functions $\bar{x}(u, v)$, $\bar{y}(u, v), \bar{z}(u, v)$ defined on the unit circle such that

$$
\begin{gathered}
\lim x_{n}(u, v)=\bar{x}(u, v), \quad \lim y_{n}(u, v)=\bar{y}(u, v), \\
\lim z_{n}(u, v)=\bar{z}(u, v)
\end{gathered}
$$


uniformly on the whole circle. Consider now the surface

$$
S: \quad x=\bar{x}(u, v), y=\bar{y}(u, v), z=\bar{z}(u, v), u^{2}+v^{2} \leqq 1 ;
$$

its boundary curve is a limit curve of the $\Gamma_{n}$, since the convergence in (6.7) is uniform on the circumference $u^{2}+v^{2}=1$, and since the $\Gamma_{n}$ have the unique limit $\Gamma$, the boundary curve of $S$ is $\Gamma$ itself. Moreover, by the semi-continuity of the Lebesgue area we have

$$
L(S) \leqq \lim \inf L\left(S_{n}\right) \leqq \lim L\left(\Pi_{n}\right)=m(\Gamma) .
$$

But since $S$ is bounded by $\Gamma$, we have

$$
L(S) \geqq a(\Gamma) ;
$$

comparing inequalities (6.8), (6.9) and (6.1), we have $L(S)=a(\Gamma)=m(\Gamma)$, which establishes equation (6.3).

By Theorem I, there exists a representation

$$
x=x(u, v), y=y(u, v), z=z(u, v), u^{2}+v^{2}=1,
$$

of $S$ for which conditions (2.1) are satisfied, and further

$$
E=G \text { and } F=0 \text { almost everywhere. }
$$

Then†

(6.12) $L(S)=\iint_{u^{2}+v<1}\left(E G-F^{2}\right)^{1 / 2} d u d v^{\circ}=\iint_{u^{2}+v<<1} \frac{1}{2}(E+G) d u d v$.

From this it follows that $x(u, v), y(u, v), z(u, v)$ are harmonic. For suppose, e.g., that $x(u, v)$ is not harmonic, and let $\xi(u, v)$ be the harmonic function having the same boundary values as $x(u, v)$. The function $\xi$ minimizesł the Dirichlet integral for the given boundary values, and is the unique minimizing function; hence for the surface

$$
\bar{S}: \quad x=\xi(u, v), y=y(u, v), z=z(u, v), u^{2}+v^{2} \leqq 1,
$$

we have $\bar{E}+\bar{G}<E+G$. Therefore

$$
\begin{aligned}
L(\bar{S}) & =\iint_{u^{2}+v^{2}<1}\left(\bar{E} \bar{G}-\bar{F}^{2}\right)^{1 / 2} d u d v \leqq \iint_{u^{2}+v^{2}<1} \frac{1}{2}(\bar{E}+\bar{G}) d u d v \\
& <\iint_{u^{2}+v^{2}<1} \frac{1}{2}(E+G) d u d v=L(S)=a(\Gamma) .
\end{aligned}
$$

$\dagger$ McShane or Morrey, loc. cit. in $\S 4$.

‡Lebesgue's proof of the minimizing property (Bulletin de la Société Mathématique de France, vol. 41 (1913); p. 48 of the Comptes Rendus) can easily be modified to show that $\xi(u, v)$ minimizes the Dirichlet integral in the class of all functions having the given boundary values and satisfying conditions (2.1). 
But $S$ is bounded by $\Gamma$, hence $L(S) \geqq a(\Gamma)$, contradicting (6.13). Hence $x(u, v), y(u, v), z(u, v)$ are harmonic.

Since $E, F, G$ are now seen to be continuous, equations (6.11) imply $E=G$, $F=0$ everywhere in the unit circle. By a theorem of Weierstrass we know that if a surface $S$ is so represented that $E=G, F=0$, the surface $S$ is minimal if and only if the functions $x, y, z$ are harmonic; these conditions being here satisfied, our surface $S$ is a minimal surface, and the theorem is proved.

Between the present solution of the problem of Plateau and that given by Radó there remains one point of difference. We have not shown that our equations (6.2) carry the circumference of the unit circle in a one-to-one way into the curve $\Gamma$. We can however very easily establish this by the same device as was used by Radó, $\dagger$ to whose work we refer the reader.

$†$ Rad6, loc. cit.; in particular, chapter 2, §3, No. 9 .

University of Chicago, Chicago, Ill. 